\title{
Authoring Tools and Virtual Environments in Intelligent Tutoring Systems: Challenges and Opportunities
}

\author{
Omar López Chávez, Luis-Felipe Rodríguez, Guillermo Salazar Lugo, \\ Luis A. Castro \\ Sonora Institute of Techonology, Department of Computer and Design, \\ México \\ omarlopch@gmail.com, luis.rodriguez@itson.edu.mx, gsalazarlugo@gmail.com, \\ luis.castro@acm.org
}

\begin{abstract}
Authoring tools in Intelligent Tutoring Systems (ITS) are designed to make it easier for tutors the use of Artificial Intelligence tools. Authoring tools allow tutors to develop adaptive, learner-centered courses that promote interaction between students and virtual tutors. This article presents a review of Virtual Reality (VR) tools for the creation of Virtual Learning Environments (VLE). In particular, we provide an analysis regarding how this kind of tools can be combined with authoring tools in ITS to achieve VLE that present to students realistic, interactive and immersive educational content. Finally, we discuss some challenges and opportunities in the design and implementation of authoring tools and tools of VR for the generation of VLE.
\end{abstract}

Keywords: Intelligent tutoring system, authoring tools, virtual reality, virtual learning environment, intelligent virtual agents.

\section{Introduction}

An Intelligent Tutoring System (ITS) acts as a private tutor of the student. An ITS can adapt to the pace of learning of each student, has a high degree of interactivity and provides the necessary feedback to each student so that they can achieve their learning goals [13]. An ITS must have autonomy to act according to the needs of the student, allowing students to apply the practice of their knowledge and skills more effectively than with traditional lessons.

The architecture implemented in such systems is basically based on three modules [27]: Expert module: It contains the description of the knowledge or behavior that represents the domain or teaching, i.e., the educational resources of a course. It allows an ITS to compare actions and choices of the student with the expert, in order to evaluate what the user knows and does not know. The student module: It assesses the information or performance of each student to determine their knowledge, reasoning ability, and perceptual skill. Pedagogical 
module: It encodes the teaching methods that are appropriate for the target domain and the student. It is the engine of execution of the adaptive system. Based on knowledge, experience and learning styles of the students, the model selects the most appropriate educational intervention.

Altogether, these modules perform specific tasks, which support actions taken by each student that is implemented through another module called interface or communication module. A general system architecture of an ITS is shown in Fig. 1.

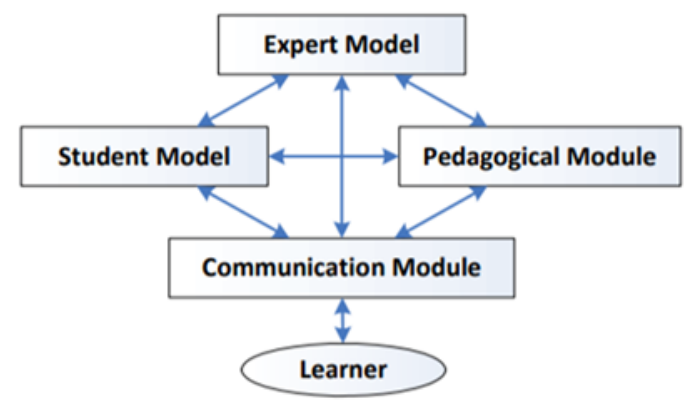

Fig. 1. Components of an ITS [27].

In ITS, authoring tools create academic content without requiring the tutor to have programming skills or technical skills. Authoring tools allow tutors to create learning content based on the activities defined in their interactive courses, learning objects (LO) and self-assessment online. Examples of such tools include RIDES [12], XAIDA [16], SIMQUEST [20], ITSB (Intelligent Tutoring System Builder)[13] and EDUCA [25].

The technology of Virtual Reality (VR) has begun to play an important role in the education process. The use of VR in ITS presents several advantages such as improved user experience through the use of interactive objects in educational settings. In particular, a Virtual Learning Environment (VLE) can be defined as a virtual environment based on a specific pedagogical model. An ITS combined with VLE tools is also called an Intelligent Virtual Environment for Training (IVETs). The interaction of the user in a VLE is usually supported by the use of pedagogical agents, which are usually implemented as Intelligent Virtual Agents (IVAs). These IVAs are embodied in virtual worlds and take the role of tutors, interacting with students and other IVAs in the VLE. In general, an IVA is capable of perceiving, adapting, and reacting to its environment. An IVA may supervise the activities of students, providing a feedback of tutoring. Course materials may be developed with these tools so that tutor-to-student interaction is more realistic. These IVAs are designed and programmed according to a process by an author. There are several tools for modeling virtual environments 
Authoring Tools and Virtual Environments in Intelligent Tutoring Systems: Challenges ...

such as SimHuman[1], ACE (Agent Common Environment) [8], OpenSimulator (OpenSim) [15] and Unity 3D [24] for interaction with the user.

Current reviews report isolated efforts regarding the development of ITS authoring tools and VR authoring tools $[18,10]$. Just a few where found where an ITS authoring tool integrates VR authoring tools capabilities [21,23]. However, authoring tools for ITS can benefit from the implementation of virtual environments as part of the teaching-learning process. For example, VLEs can be created to provide more immersive experiences by allowing the user to be guided by IVAs; interact with other students, a teacher or human tutors using avatars in a virtual world and finally allowing the user to interact with simulated scenarios where conditions are difficult to replicate in reality or involves high costs.

In general, authoring tools lack mechanisms to create academic content based on virtual environments. However, as mentioned above, the use of VLEs as part of the learning process presents several benefits. In this article we present a review of authoring tools for ITSs and tools for the development of VLEs. In particular, we analyze how authoring tools can take advantage of existing tools designed to create virtual environments in order to allow tutors to generate more realistic and immersive academic content, and more realistic and immersive interaction between IVAs and students.

\section{Authoring Tools in Intelligent Tutoring Systems}

A wide variety of authoring tools for ITSs have emerged over the last 25 years. Some of them have been discontinued, but a few authoring toolsets continue to evolve. Each of these tools has different scope (e.g., authoring for model-tracing, agent-based, or dialogue-based tutors) and a different set of learning theories (e.g., Component Display Theory and Cognitive Load Theory) that drive their design [22].

Currently, there are many authoring tools for ITS. Table 1 shows some of these tools classified on the basis of their design goals. In category Teachercentered, authoring tools are focused on pedagogy and focuses on how to teach. In category Student-centered, tools are designed to perform in learning environments in which students can develop skills by practicing and receiving feedback $[12]$.

Tutors through the ITS Authoring Tools are able to design workflows, constraints, content, adaptability rules, which allow the intelligent agent to perform recommendations, counseling and evaluations as a human tutor. A brief description of the most important of them are shown in Table 2 as a comparison.

\section{Virtual Reality in Education}

Since the 90's, VR technology has been used in educational desktop applications where students interacted with 3D simulations [3]. In the last 15 years, VR in VLEs has gained great importance in the process of teaching and learning. For 
Table 1. ITS authoring tools by category.

\begin{tabular}{lll}
\hline Category & $\begin{array}{l}\text { Authoring } \\
\text { tools }\end{array}$ & Focus \\
\hline Curriculum & DOCENT [26], & \\
Sequencing and Planning & IDE [17], ISD Expert, Expert CML [7] & Teacher-centered \\
Tutoring & Eon [5], & (teaching process) \\
Strategies & GTE, REDEEM [2] & \\
Multiple & CREAM-Tools, \\
Knowledge Types & DNA, ID-Expert [11], IRIS, XAIDA [16] \\
Intelligent/adaptive & CALAT [9], & \\
Hypermedia & GETMAS, InterBook, MetaLinks & \\
\hline Device & DIAG, & \\
Simulation and Equipment Training & RIDES [12], SIMQUEST [20], XAIDA [16] Student-centered \\
Domain & Demonstr8, & (learning process) \\
Expert System & D3 Trainer, Training Express & \\
Special & IDLE-Tool/IMap, & \\
Purpose & LAT & \\
\hline
\end{tabular}

example, using VLE with VR simulation, students visited places or recreated the history [25].

The teaching design based on VR technology has enabled teachers to use tools such as Unity (see Fig. 2) to recreate VLE from real places where students can participate in realistic activities in that environment. For example, the tool Unity 3D can generate virtual and VR environments for education through virtual characters and 3D components with animations that can be used by an agent module that implements a tutor.

OpenSimulator (see Fig. 3) can generate alternative virtual worlds for education where tutors create a space with students to design laboratories and objects that are used to learn the content and skills. Likewise, OpenScenegraph is a tool designed to facilitate the creation of virtual reality worlds where tutors are virtually represented by IVAs and students are represented by avatars, as well as virtual objects that can interact in this VLE.

In a VLE, it is possible to have IVAs that can take multiple roles. For example, an IVA can be designed as an Expert Agent, a Tutoring Agent, a Communication Agent, a Student Modeling Agent or a World Agent. The definition of the role of each IVA depends on different scenarios where having an interaction with the user is needed. IVAs allow a more human-like interaction with users. They allow applications to have more expressive and emotional content since an IVA can be based on 3D models that closely match a person's face and body. The expression of emotions in IVAs can increase user's creativity by creating an illusion of life. A VLE that integrates IVAs promotes a more natural interaction between the virtual tutor and the user, so that students will have a more effective communication.

The creation of a virtual environment requires a script or design of the elements that inhabit that environment, such as 3D objects, agents, avatars. Depending on the environment, IVAs are added for IVA-user and IVA-IVA interaction. Currently, there are tools for the design of virtual environments such as Second Life (SL) [19], OpenSimulator (OpenSim)[15], OpenScenegraph[14], Agent Common Environment (ACE)[8], SimHuman[1] and Unity[24]. Table 3 
Authoring Tools and Virtual Environments in Intelligent Tutoring Systems: Challenges ...

Table 2. Authoring tools in intelligent tutoring systems.

\begin{tabular}{ll}
\hline Tools & Functionality \\
RIDES[12] & Helps the author to visualize the \\
& relationships between the elements of the curriculum (such as topics, courses, \\
& concepts, and procedures). \\
& It allows to develop simulations. \\
& Offers \\
XAIDA [16] & training in maintenance in four areas: the physical characteristics of a \\
& device, theory of operation, operation and maintenance and troubleshooting \\
& procedures. \\
& Develops \\
SIMQUEST[20] & systems of simulation-based learning", the author creates the \\
& simulation model, the interface of the student, instructional design and the \\
& environment. \\
& Designed \\
& and developed for teachers in ITS building in multidisciplinary areas. The \\
& author can add materials of the course, questions and answers, etc. As well as \\
& modules with their objectives, evaluations, etc. \\
& Develop \\
ITSB [13] & Adaptive learning material in a Web 2.0 collaborative learning environment. \\
& Another feature is the export the material to content SCORM (Sharable Content \\
& Object Reference Model) for use in online platforms. \\
& Developed \\
to support problem-based task domain. Requires familiarity with the Java Expert \\
System Shell (JESS) production rule language. CTAT is currently available as \\
binary (executable) code. \\
Strives \\
for authoring toolsets that are easy to access and use, and support authoring \\
in multiple task domains (cognitive, affective, psychomotor, and social). Its \\
vision is for a shell tutor or architecture where a variety of ITSs can support \\
training in a variety of task domains. \\
CTAT[4]
\end{tabular}

shows some of the tools for the generation of most commonly used virtual environments.

Table 3. Comparison of VR tools.

\begin{tabular}{|c|c|c|c|c|c|c|}
\hline $\begin{array}{ll}\text { Characteristics } & \text { Tools } \\
\end{array}$ & $\mathrm{SL}$ & penSim & OpenScenegraph & $\mathrm{ACE}$ & SimHuman & Unity \\
\hline $\begin{array}{l}\text { Access } \\
\text { to accounts }\end{array}$ & $\mathrm{X}$ & $\mathrm{X}$ & & $\mathrm{X}$ & & \\
\hline $\begin{array}{l}\text { 3D } \\
\text { design. Object Scheduler }\end{array}$ & $\mathrm{X}$ & $\mathrm{X}$ & $\mathrm{X}$ & $\mathrm{X}$ & $\mathrm{X}$ & $\mathrm{X}$ \\
\hline $\begin{array}{l}\text { Virtual } \\
\text { Worlds }\end{array}$ & $\mathrm{X}$ & $\mathrm{X}$ & $\mathrm{X}$ & $\mathrm{X}$ & $\mathrm{X}$ & $\mathrm{X}$ \\
\hline $\begin{array}{l}\text { Intelligent } \\
\text { Virtual Agents (IVAs) }\end{array}$ & $\mathrm{X}$ & $\mathrm{X}$ & & $\mathrm{X}$ & $\mathrm{X}$ & $\mathrm{X}$ \\
\hline $\begin{array}{l}\text { Interactive } \\
\text { Script }\end{array}$ & & $\mathrm{X}$ & $\mathrm{X}$ & $\mathrm{X}$ & & $\mathrm{X}$ \\
\hline $\begin{array}{l}\text { Programming } \\
\text { Knowledge }\end{array}$ & & $\mathrm{X}$ & $\mathrm{X}$ & $\mathrm{X}$ & $\mathrm{X}$ & $\mathrm{X}$ \\
\hline
\end{tabular}

\section{Challenges and Opportunities}

Through the literature review it has been identified tree scenarios where VR and ITS converge: Virtual Learning Environments, Simulations, and Intelligent 
Omar López Chávez, Luis-Felipe Rodríguez, Guillermo Salazar Lugo, Luis A. Castro

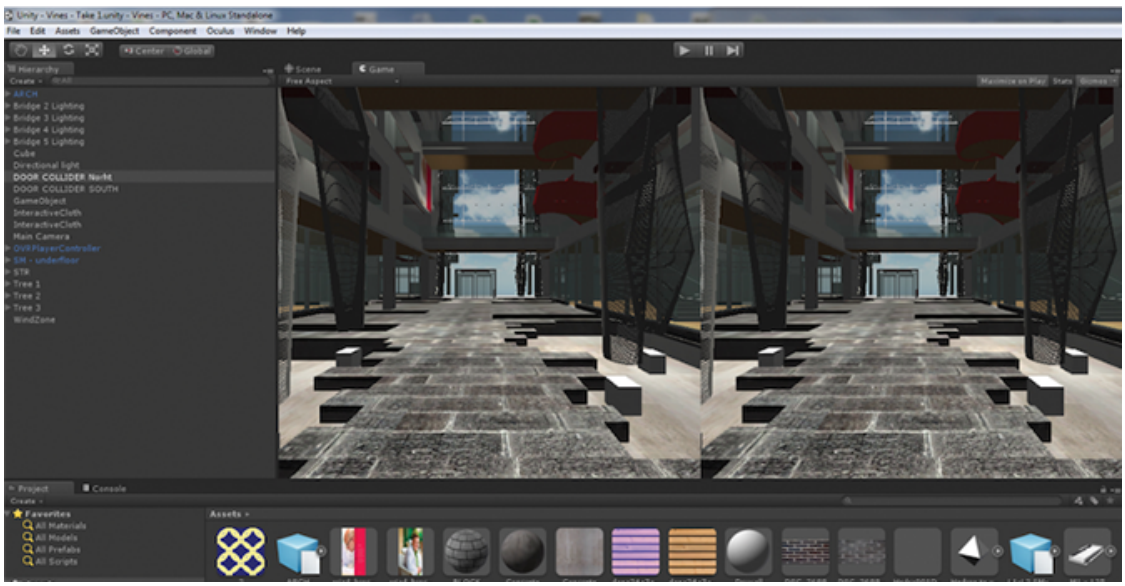

Fig. 2. Screen of a unity implementation [24].

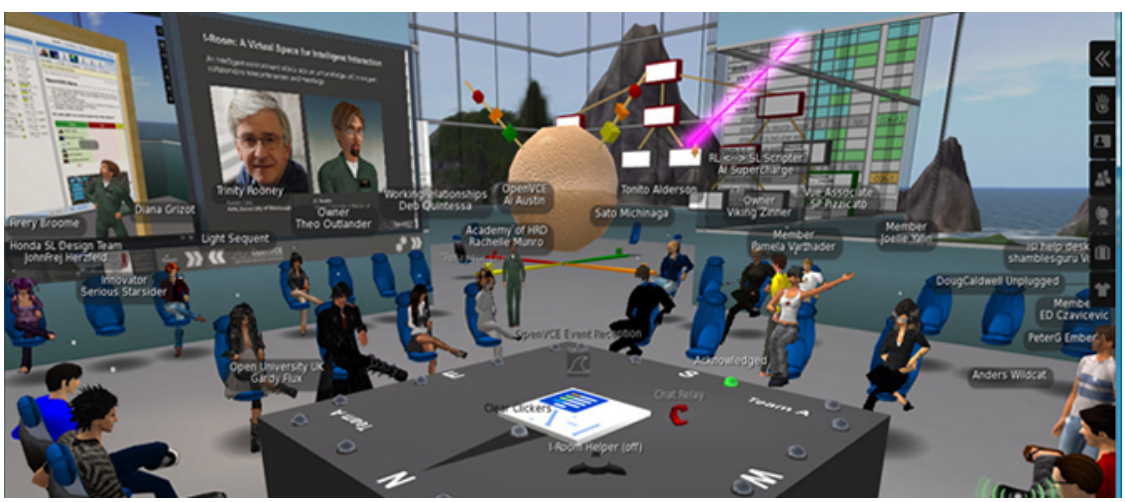

Fig. 3. OpenSimulator 3D virtual world environment[15].

Virtual Agents. Furthermore, we visualize some major challenges and opportunities:

- Traditional ITS are focused on tutoring students in online courses by simulating the behavior of a human tutor and adapting to the student's behavior in order to offer help when required. In order to achieve more realistic behaviors in ITS, it is necessary to include IVAs. However, IVAs should become more autonomous, capable of learning, have more natural and adaptive interactions according to the dynamics of the conversation with the user and changes in the environment. Also, providing a realistic representation of a human tutor to address some aspects related to factors that influence on learning, e.g. motivation, stress and feedback.

- The development of virtual environments with VR requires the simulation of virtual worlds inhabited by IVAs, where learners and human tutors are 
Authoring Tools and Virtual Environments in Intelligent Tutoring Systems: Challenges ...

capable of interacting like if they were in the same place.

- The overlap of the capabilities of VR and ITS could be addressed through the integration of VR authoring tools and ITS authoring tools providing ways for developing, integrating and reusing Virtual Environments, Simulations and Intelligent Virtual Agents.

- Currently one of the opportunities for developers of E-Learning platforms is the creation of VR environments capable of adapting to educational strategies where a more affective learning experience is implemented.

- Finally, it is necessary to enable the tutor to take advantage of VR on e-learning environments through ITS-VR Authoring tools without requiring technical knowledge on VR design and development to create IVAs, Simulations and Learning Virtual Worlds.

\section{Conclusion}

This paper analyzed the challenges and opportunities of combining ITS Authoring tools with Virtual Reality tools for creating academic content. We found that usually the development of simulations using VR to model explanations is highly expensive or requires complex scenarios to be replicated. Nevertheless, the literature review showed a great variety of ITS Authoring tools that allow the creation of educational content. We identified some of such tools aimed at helping the author to create simulation models for instructional design and virtual environments, which are based on free software. As future work, we are planning to integrate VR authoring tools within ITS authoring tools to improve the creation of intelligent tutors in realistic, interactive and immersive content.

\section{References}

1. Simhuman: A platform for real-time virtual agents with planning capabilities. In: Vosinakis, S., Panayiotopoulos, T. (eds.) Proceedings of the Third International Workshop on Intelligent Virtual Agents (2001)

2. Ainsworth, S., Major, N., Grimshaw, S.K., Hayes, M., Underwood, J.D., Williams, B., Wood, D.J.: Redeem: Simple intelligent tutoring systems from usable tools. pp. 205-232. In T. Murray and S.Blessing and S. Ainsworth (Eds.), Amsterdam: Kluwer Academic Publishers (2003)

3. Cassell, J., Gill, A.J., Tepper, P.A.: Coordination in conversation and rapport. In: In Proceedings of the workshop on Embodied Language Processing. pp. 41-50. As-sociation for Computational Linguistics (2007)

4. CTAT: Cognitive tutor agent tools. http://ctat.pact.cs.cmu.edu/ (2017)

5. EON: Eon reality inc. https://www.eonreality.com/ create-vibrant-interactive-3d-environments-2/ (2017)

6. GIFT: Generalized intelligent framework for tutoring. https://www . gifttutoring.org (2017)

7. Jones, M., Wipond, K.: Intelligent Environments for Curriculum and Course Development. Teaching Knowledge and Intelligent Tutoring. Norwood, NJ: Ablex. (2015) 
8. Kallmann, M., Thalmann, D.: A behavioral interface to simulate agent-object interactions in real-time. In: Proceedings of Computer Animation 99, IEEE Computer Society Press. pp. 138-146. Geneva (1999)

9. Kiyama, M., Ishiuchi, S., Ikeda, K., Tsujimoto, M., Fukuhara, Y.: Authoring methods for the web-based intelligent cai system calat and its application to telecommunications service. In: the Proceedings of AAAI-97: Conference of the American Association for Artificial Intelligence, Providence, RI.- cited (1997)

10. Marcus, A., Abromowitz, S., Abulkhair, M.: Design, user experience, and usability. In: User Experience in Novel Technological Environments: Second Int. Conf., DUXU. pp. 21-26. Springer (2013)

11. Merrill, M.: An expert system for instructional design. IEEE-Expert 2(2), 25-37 (1987)

12. Munroe, A., Pizzini, Q., Towne, D., Wogulis, J., Coller, L. (eds.): Authoring procedural training by direct manipulation. USC Behavioral Technology Laboratories Working Paper WP94-3, University of Southern California, Redondo Beach, CA. (1994)

13. Naser, S.S.A.: Predicting learners performance using artificial neural networks in linear programming intelligent tutoring system. International Artificial Intelligence \& Applications 3(2), 65-73 (2012)

14. OpenSceneGraph: http://www.openscenegraph.org/ (2017)

15. OpenSimulator: http://opensimulator.org/ (2017)

16. Patricia, Y.H., Henry, M.H., Carol, L.R.: Four easy pieces: development systems for knowledge-based generative instruction. International Journal of J. Artificial Intelligence in Education (IJAIED) 10, 1-45 (1999)

17. Pirolli, P., Russell, D.: Instructional design environment: technology to support design problem solving. Instructional Science 19(2), 121-144 (1991)

18. Schmorrow, D.D., Reeves, L.M.: Foundations of augmented cognition. Springer (2005)

19. SecondLife: http://secondlife.com (2017)

20. Simquest: An alternative way of learning. http://www.simquest.nl (2017)

21. Sottilare, R.A., Brawner, K.W., Goldberg, B.S., Holden, H.K.: The generalized intelligent framework for tutoring (gift) (2012)

22. Sottilare, R.A., Graesser, A., Hu, X., Holden, H.: Design Recommendations for Intelligent Tutoring Systems: Volume 1-Learner Modeling, vol. 1. US Army Research Laboratory (2013)

23. Trausan-Matu, S., Boyer, K., Crosby, M., Panourgia, K.: Intelligent Tutoring Systems. Springer International Publishing (2014)

24. Unity3D: https://unity3d.com/es (2017)

25. Winn, W., Windschitl, M.: Learning science in virtual environments: the interplay of theory and experience. Themes Educ 1(4), 373-389 (2000)

26. Winne, P.H., Kramer, L.L.: Representing and inferencing with knowledge about teaching: Docent - an artificially intelligent planning system for teachers. In: Proceedings of Intelligent Tutoring System (ITS-88). pp. 7-15. Montreal (1988)

27. Zarandi, M.H.F., Khademian, M., Minaei-Bidgoli, B., Türkşen, I.B.: A fuzzy expert system architecture for intelligent tutoring systems: A cognitive mapping approach. Journal of Intelligent Learning Systems and Applications 4(01), 29-40 (2012) 